\title{
QTc interval prolongation and cirrhosis severity in non- alcoholic patients
}

\author{
Amin Saeidinia ${ }^{1,2}$, Arsalan Salari ${ }^{2}$, Afshin Shafaghi ${ }^{3 *}$ and Mahshid Ofoghi ${ }^{1}$ \\ ${ }^{1}$ Mashhad University of Medical Sciences, Mashhad, Iran \\ ${ }^{2}$ Guilan University of Medical Sciences, Rasht, Iran \\ ${ }^{3}$ Gastrointestinal and Liver Diseases Research Center (GLDRC), Guilan University of Medical Sciences (GUMS), Razi Hospital, Rasht, Iran
}

\begin{abstract}
Introduction: Chronic liver disease is a major health problem and important causes of mortality and morbidity. Cirrhosis is associated with cardiovascular abnormalities. QT interval prolongation is one of the electrophysiological indicators of cirrhotic cardiomyopathy. So in this study we evaluated the QTc interval prolongation and cirrhosis severity in non-alcoholic patients admitted in Razi hospital in Rasht during 2011-2012.

Material \& methods: This survey was conducted as a cross-sectional descriptive study. A 12-lead surface ECG and echocardiographic study was obtained from all subjects. A 12-lead electrocardiogram recorded at $50 \mathrm{~mm} /$ second to measure the QT interval. The mean QT interval in every 12 leads was documented for each patient. The QT corrected for RR (QTc) was calculated. The quantitative and qualitative data were analyzed by Chi-square, ANOVA, Linear regression test and Fisher's exact tests.

Results: The mean of age was $52.78 \pm 15.2$. Sixty-three persons were male, and others were female. The most common cause of cirrhosis in our study population was Hepatitis $\mathrm{C}$ virus. Prolonged QTc interval was seen in $48 \%$ of subjects. There was no significant correlation between age and mean of laboratory data and QTc prolongation $(\mathrm{P}>0.05)$. There was no significant correlation between causes of cirrhosis and QTc prolongation $(\mathrm{P}>0.05)$. There was significant correlation between severity of cirrhosis and QTc $(\mathrm{P}=0.020)$.
\end{abstract}

Conclusion: Majority of patients with cirrhosis showed QTc interval prolongation, which related significantly with worsening the severity of the disease, regardless of the etiology of cirrhosis.

\section{Introduction}

Chronic liver disease is a major health problem and causes of mortality and morbidity. Almost 30,000 patients each are reported to develop cirrhosis in United States [1]. Cirrhosis is a hepatic disease that presents individuals aged 50-60 age group, typically [2,3]. A hyperdynamic circulation, which is manifested as high cardiac output, decreased systemic vascular resistance and widespread arterial vasodilatation at first in the cirrhosis $[4,5]$. Based on previous many studies traditionally, cirrhosis is associated with cardiovascular abnormalities [6,7].

Cirrhotic cardiomyopathy is the term used to describe a collection of characters expressive of abnormal heart structure and function in patients with cirrhosis $[8,9]$. The term "cirrhotic cardiomyopathy" is generally defined by the following clinical criteria: 1) baseline increased cardiac output but blunted ventricular response to stimuli, 2) systolic and/or diastolic dysfunction, 3) absence of overt left ventricular failure at rest, 4) electrophysiological abnormalities including prolonged QT interval on electrocardiography and chronotropic incompetence [1012].

The QT interval in the electrocardiography reflects ventricular repolarization and its prolongation provides substrate for ventricular arrhythmias. QT interval prolongation is one of the electrophysiological indicators of cirrhotic cardiomyopathy $[13,14]$. It is assumed that this abnormality occurs due to cardiotoxins reaching the heart due to portosystemic shunting. QT interval is affected by heart rate. Few studies have shown prolonged QT interval to be associated with severity of liver disease [15]. In this setting, the QT interval is further prolonged by transjugular intrahepatic porto-systemic shunt and is shortened or normalized by acute beta-blockade6 and liver transplantation. However, the true clinical meaning of the QT interval remains unsettled [16-19].

Therefore, in this study we evaluated the QTc interval prolongation and cirrhosis severity in non-alcoholic patients admitted in Razi hospital in Rasht during 2011-2012.

\section{Material \& methods}

This is a cross sectional descriptive study. Sampling was performed as census in all cirrhotic patients referred and admitted in the educational Razi hospital in Rasht since 2011-2012. We published previously a part of this project.

*Correspondence to: Afshin Shafaghi, Division of Gastroenterology \& Hepatology, Department of Gastroenterology, Gastrointestinal and Liver Diseases Research Center (GLDRC), Guilan University of Medical Sciences (GUMS), Razi Hospital, Sardar-Jangal Avenue, Rasht, Iran, Tel: +981313223444; E-mail: drafshinshafaghi@gmail.com

Key words: QT interval prolongation, cirrhosis severity, non-alcoholic cirrhosis

Received: April 29, 2020; Accepted: June 05, 2020; Published: June 08, 2020 


\section{Patients selection}

In this study, one hundred patients with definitive diagnosis of cirrhosis referred to our unit over the 2 years were enrolled consecutively. The diagnosis of cirrhosis was based on histology and/or clinical, biochemical and imaging features. Exclusion criteria were the patients with consumption of alcohol (>90 cc/day). None of the patients were receiving drugs affecting ventricular repolarization and if betablockers were given for portal hypertension, the drug was withdrawn for a week before calculating measurements.

For all patients clinical evaluation, and the Child-Pugh score was calculated. The study protocol was approved by the ethics committee of Guilan University of medical sciences by the Helsinki declaration guidelines. Informed consent in writing was obtained by each subject.

\section{Electrocardiography}

A 12-lead electrocardiogram was recorded at $50 \mathrm{~mm} / \mathrm{second} \mathrm{to}$ measure the QT interval. The QT intervals of three consecutive sinus beats were manually measured by means of a caliper in each of the 12 leads where the end of the $\mathrm{T}$ wave could be reliably identified, and a mean QT interval per lead was calculated.

Mean QT interval in every 12 leads was documented for each patient in maximum. Measurements were made by an observer not aware of the patients' characteristics. The QT corrected for RR (QTc) was calculated by the applied correction formulas [20].

\section{Statistical methods}

SPSS for Windows, version 16 was used for data entry. Kolmogorov-Smirnov test was performed for data distribution. The quantitative and qualitative data were analyzed by Chi-square, ANOVA, Linear regression test and Fisher's exact test. Continuous variables are presented as mean \pm standard deviation (SD); categorical variables are presented as percentages. $\mathrm{P}_{\text {value }}<0.05$ was considered as significant.

\section{Results}

Mean age of the patients was $52.78 \pm 15.2$ years old. 63 subjects were male $(63 \%)$ and 37 of them were female $(37 \%)$. The most common cause of cirrhosis in our study population was Hepatitis $\mathrm{C}$ virus. The mean of duration of disease in all cases is $2.83 \pm 4.02$ years.

In Table 1 severity of cirrhosis has been shown based upon ChildPugh classification. Most of patients were in severe type of cirrhosis (40\%).

Table 2 shows the range and means values of some laboratory results of studied patients.

There was no significant correlation between age and mean of laboratory data and QTc prolongation $(\mathrm{P}>0.05)$. QTc prolongation was more in male patients significantly $(\mathrm{P}=0.29)$.

The mean of QTc values was $461.69 \pm 39.40 \mathrm{~ms}$. Frequency of normal or prolongation QTc has been shown in Table 3 in age groups. There was no significant correlation between causes of cirrhosis and QTc prolongation $(\mathrm{P}>0.05)$.

Prolonged QTc interval was seen in $48 \%$ of subjects. The age group of 40-60 years had the most frequency of QTc prolongation (24, $48 \%)$. There was a significant correlation between QTc prolongation and gender $(\mathrm{P}=0.035)$ and it was more common in male. There was no meaningful correlation between existence of QTc prolongation and duration of disease $(\mathrm{P}=0.178)$ and age $(\mathrm{P}=0.948)$. There was significant relation between the mean of QTc and Child-Pugh grade $(\mathrm{P}=0.020)$.

Table 4 shows frequency of QTc prolongation in different class of cirrhosis severity. There was no significant correlation between severity of cirrhosis and QTc prolongation ( $\mathrm{P}=0.679)$ (Figure 1).

Gender $(\mathrm{P}=0.005)$, duration of disease $(\mathrm{P}=0.001)$ and PAP (pulmonary artery pressure) $(\mathrm{P}=0.021)$ can significantly predict the $\mathrm{QT}$ prolongation in our population.

\begin{tabular}{|c|c|c|}
\hline \multicolumn{3}{|c|}{ Child-Pugh classification } \\
\hline Child A & Mild & $22(22 \%)$ \\
\hline Child B & Moderate & $38(38 \%)$ \\
\hline Child C & Severe & $40(40 \%)$ \\
\hline
\end{tabular}

Table 2. Range and mean values of some laboratory results in the hospitalized patients

\begin{tabular}{|c|c|c|}
\hline & Mean & Standard Deviation \\
\hline Serum Cr & 1.03 & 0.66 \\
\hline Table 1. Setermy ¿á cirrhosis & 1.39 & 5.24 \\
\hline Serum K & 4.27 & 0.54 \\
\hline Serum Ca & 8.17 & 0.65 \\
\hline
\end{tabular}

Table 3. Distribution of QTc prolongation in various age groups

\begin{tabular}{|c|c|c|c|}
\hline \multirow{2}{*}{ Age Group } & \multicolumn{2}{|c|}{ QTe interval } & \multirow{2}{*}{ Total } \\
\cline { 2 - 4 } & Normal & Long & 22 \\
\hline$<40$ & 12 & 10 & 48 \\
\hline $40-60$ & 24 & 24 & 30 \\
\hline$>60$ & 16 & 14 & 100 \\
\hline Total & 52 & 48 & \\
\hline
\end{tabular}

Table 4. Severity of cirrhosis and QTc Interval

\begin{tabular}{|c|c|c|c|}
\hline \multirow{2}{*}{ Child-Pugh } & \multicolumn{2}{|c|}{ QTe Interval } & \multirow{2}{*}{ Total } \\
\cline { 2 - 3 } & Normal & Long & 22 \\
\hline Mild & 13 & 9 & 38 \\
\hline Moderate & 20 & 18 & 40 \\
\hline Severe & 19 & 21 & 100 \\
\hline Total & 52 & 48 & \\
\hline
\end{tabular}

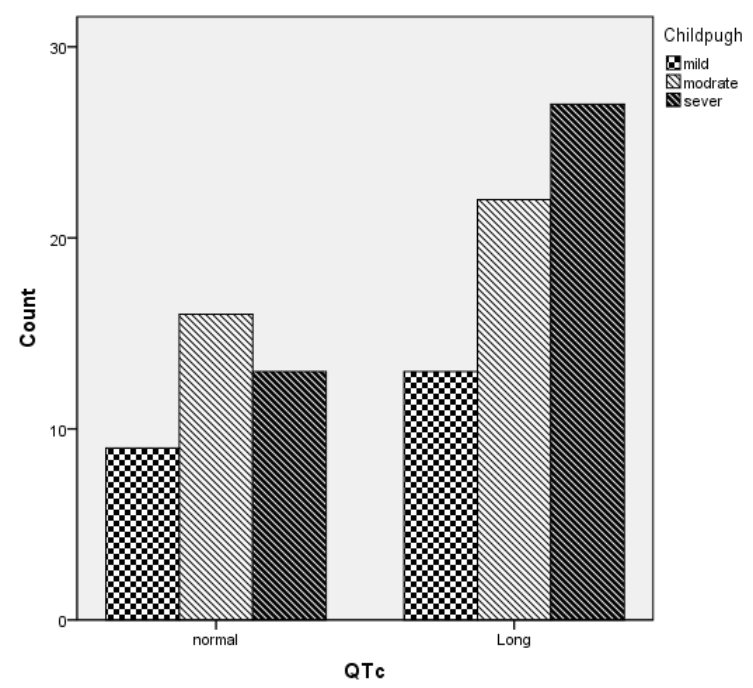

Figure 1. Qtc prolongation and cirrhosis severity 


\section{Discussion}

Previous studies showed the prolongation of the QT interval in cirrhosis patients [17,21-23]. Cardiomyopathy due to cirrhosis is a wellknown problem and QT interval prolongation is an indicator of cardiac dysfunction. In about $50 \%$ of cirrhotic patients, long QT interval is appeared that can affect cardiac morbidity and mortality [24]. The pathogenesis of cirrhotic cardiomyopathy includes diminished betaadrenergic receptor signal transduction; cardiomyocyte cellular plasma membrane dysfunction; and increased activity or levels of cardiodepressant substances such as cytokines, endogenous cannabinoids, and nitric oxide. Although cirrhotic cardiomyopathy is usually clinically silent, or not very severe, overt heart failure can be triggered by factors such as liver transplantation or transjugular intrahepatic portosystemic shunt insertion in these patients [25]. A frequent occurrence of QTc interval prolongation has been found in patients with alcoholic liver disease and candidates for liver transplantation because of advanced cirrhosis $[19,25]$. This abnormality is not related to the etiology of cirrhosis. In this study, we evaluated the prevalence of QTc interval in cirrhosis patients and its relationship with severity of cirrhosis in nonalcoholic cirrhotic patients.

The most common group affected by cirrhosis in our study was 40 60 years old group (48\%). Similarly to our study Bhatti et al. [21] and Genovesi et al. showed in their study that most of the patients were $>45$ years of age $(86.1 \%)$, with the majority being in the $45-60$ year age group (51.2\%), indicating an increased prevalence of cirrhosis in higher age groups, which is understandable considering the long natural history of cirrhosis; it may take up to 30 years from the onset of infection to development of cirrhosis [26]. However, the patient age two other studies, Zuberi et al. reported a much lower mean age of 34.4 years in their study population [27].

Majority of our patients were in B (38\%) and C (40\%) Child-Pugh grade. This was not unexpected because the samples were taken mostly from the inpatient department, and Child Pugh Grade A patients are admitted less frequently than grade B or C patients. Zuberi et al. and Bhatti et al. also described a similarly distribution of patients with most of them being in Child Pugh Grade B and C [21,27].

Our results showed that the mean of QTc values was $461.69 \pm 39.40$ ms. It was similar to other studies and was comparable with them. Bernardi et al. also conducted a similar investigation and reported a mean QTc of $440 \pm 3.2 \mathrm{~ms}$ [17]. Li et al. demonstrated a mean QTc of $421 \pm 38 \mathrm{~ms}$ in their study [28]. A study by Zuberi et al. demonstrated a mean QTc of $438 \mathrm{~ms} \pm 15 \mathrm{~ms}$ [27].

The most common cause of cirrhosis was HCV virus in our study which is adopted with Zambruni's study results [20] and Bernardi et al. study [17]. But in Zamirian et al. HBV was the most cause of cirrhosis [29].

Our study showed that prolonged QTc was seen in $48 \%$ of patients. Our result was closed to the results of Bernardi et al. study in which they showed the frequency of QTc prolongation to be 46.2\% [17]. Also it was similar to Li et al. which was $46.93 \%$ [28]; Bal et al. which was 56\% [16]; Kosar et al. that was 32\% [30] and Nasr et al. that was $45 \%$ [31]. But it was differ from Zuberi et al. study with 19.2\% [27]; Bhatti et al. [21] study with $24.7 \%$ and Lossnitzer et al. [32] with $25 \%$. These differences may be related to other confounding factors such as electrolyte disturbances, concomitant cardiac problems, or use of QTc prolonging drugs in different studies.
In our study QTc prolongation was more in male patients significantly $(\mathrm{P}=0.29)$. It was in contrast with other studies $[16,28,30]$. however, this finding is similar to Bernardi's et al. study [17] and it can confirmed the hypothesis that suggested such an androgen deficiency may potentially account for QTc prolongation in male patients with cirrhosis [33] which is need to evaluated more in our population.

There was no meaningful correlation between age and QTc interval and age which is adopted with Genovesi et al. study that reported ECG parameters had no relation with age [34]. The pathogenesis of prolonged QTc in liver disease remains unexplained. QT interval is the time between the earliest activation of ventricular myocardium to the end of latest ventricular repolarization. Therefore, the QT interval can be prolonged either because the activation is slowed or the complex process of repolarization is prolonged [22].

In our study with increase of severity of cirrhosis frequency of long QTc was elevated. Although there was no significant relationship between presence of prolonged QT interval and severity of liver disease $(\mathrm{P}>0.05)$, but it was significant relationship between the mean of QTc with the worsening of the Child Pugh Score $(\mathrm{P}=0.020)$. Our results were similar to Bernardi et al., Li et al. and Bhatti et al. studies $[17,21,28]$. Genovesi et al. also showed significant correlation between QTc and Child Pugh in his study [35]. Also in the study of Puthumana et al. this correlation was seen [22]. However, Nasr et al. showed the reverse results [31]. It is necessary to say that majority of patients in our study consumed beta blocker drugs for preventing esophageal varice bleeding that can shorten QT interval but is not affected QTc interval. On the other hand, port systemic shunt due to portal hypertension resulted in entering cardio-active agents to systemic circle that can affect cardiac repolarization. These results indicated a direct relationship between the stage of cirrhosis and the development of cirrhotic cardiomyopathy. In other words, the more advanced the cirrhosis, the greater the chances of developing cirrhotic cardiomyopathy and the associated symptoms.

There was no significant correlation between cirrhosis etiologies and QTc prolongation $(\mathrm{P}>0.05)$. This finding is similar to other studies $[17,19$. QTc is affected by a number of factors other than cirrhosis itself, such as electrolyte disturbances and certain drugs and it is recommended that the changes in cardiac function happened regardless of the etiology of cirrhosis.

\section{Conclusion}

Cardiac dysfunction is a common complication of advanced cirrhosis that can make variety of disturbance, specially QT interval prolongation and diastolic dysfunction. In conclusion, we showed that more than half of the patients with cirrhosis showed prolongation in QTc interval, which is paralleled the severity of the disease, regardless of the etiology of cirrhosis. Patients with cirrhosis with QTc prolongation have a worse prognosis, as compared to patients with normal. QTc duration. Finally because of prevalence of QTc interval disturbance it is recommended that routine ECG be done for cirrhotic patients. Also doing comprehensive study for evaluating more laboratory data and following variables in cirrhotic patients to achieving best predicting factors in this region are recommended. Also we showed that Gender, duration of disease and PAP in echocardiography can significantly predict the QT prolongation in cirrhotic population.

\section{Conflict of interests}

The authors declare that they have no conflict of interests. 


\section{References}

1. Bell BP, Manos MM, Zaman A, Terrault N, Thomas A, et al. (2008) The epidemiology of newly diagnosed chronic liver disease in gastroenterology practices in the United States: results from population-based surveillance. The American Journal of Gastroenterology 103: 2727-2736.

2. Karasu Z, Mindikoglu AL, Van Thiel DH (2004) Cardiovascular problems in cirrhotic patients. The Turkish Journal of Gastroenterology 5: 126-132.

3. Valeriano V, Funaro S, Lionetti R, Riggio O, Pulcinelli G, et al. (2000) Modification of cardiac function in cirrhotic patients with and without ascites. The American Journal of Gastroenterology 95: 3200-3205.

4. Kowalski HJ, Abelmann WH (1953) The cardiac output at rest in Laennec's cirrhosis The Journal of Clinical Investigation 32: 1025-1033.

5. Ziada D, Gaber R, Kotb N, Ghazy M, Nagy H (2011) Predictive value of N-terminal pro B-type natriuretic peptide in tissue doppler-diagnosed cirrhotic cardiomyopathy. Heart Mirror Journal 5: 264-270.

6. Moller S, Henriksen J (2005) Ascites and renal dysfunction in liver disease. Gines P, Arroyo V, Rodes J, Schrier R, editors. Malden: Blackwell.

7. Moller S, Henriksen JH (2008) Cardiovascular complications of cirrhosis. Gut 57: 268278 .

8. Finucci G, Desideri A, Sacerdoti D, Bolognesi M, Merkel C, et al. (1996) Left ventricular diastolic function in liver cirrhosis. Scandinavian Journal of Gastroenterology 31: 279-284.

9. Pozzi M, Carugo S, Boari G, Pecci V, de Ceglia S, et al. (1997) Evidence of functional and structural cardiac abnormalities in cirrhotic patients with and without ascites. Hepatology 26: 1131-1137.

10. Gaskari SA, Honar H, Lee SS (2006) Therapy insight: Cirrhotic cardiomyopathy Nature Clinical Practice Gastroenterology \& Hepatology 3: 329-337.

11. Iwakiri Y, Groszmann RJ (2006) The hyperdynamic circulation of chronic liver diseases: from the patient to the molecule. Hepatology 43: S121-S131.

12. Liu H, Song D, Lee SS (2002) Cirrhotic cardiomyopathy. Gastroenterologie clinique et biologique 26: 842-847.

13. Kass RS, Moss AJ (2003) Long QT syndrome: novel insights into the mechanisms of cardiac arrhythmias. The Journal of Clinical Investigation 112: 810-815.

14. Wood A (2004) Drug-induced prolongation of the QT interval. N Engl J Med 350 1013-1022.

15. Trevisani F, Merli M, Savelli F, Valeriano V, Zambruni A, et al. (2003) QT interval in patients with non-cirrhotic portal hypertension and in cirrhotic patients treated with transjugular intrahepatic porto-systemic shunt. Journal of Hepatology 38: 461-467.

16. Bal JS, Thuluvath PJ (2003) Prolongation of QTc interval: relationship with etiology and severity of liver disease, mortality and liver transplantation. Liver International 23: $243-248$.

17. Bernardi M, Calandra S, Colantoni A, Trevisani F, Raimondo ML, et al. (1998) Q-T interval prolongation in cirrhosis: prevalence, relationship with severity, and etiology of the disease and possible pathogenetic factors. Hepatology 27: 28-34.

18. Henriksen JH, Bendtsen F, Hansen EF, Moller S (2004) Acute non-selective betaadrenergic blockade reduces prolonged frequency-adjusted Q-T interval (QTc) in patients with cirrhosis. Journal of Hepatology 40: 239-246.
19. Mohamed R, Forsey PR, Davies MK, Neuberger JM (1996) Effect of liver transplantation on QT interval prolongation and autonomic dysfunction in end-stage liver disease. Hepatology 23: 1128-1134.

20. Zambruni A, Di Micoli A, Lubisco A, Domenicali M, Trevisani F, et al. (2007) QT interval correction in patients with cirrhosis. Journal of Cardiovascular Electrophysiology 18: 77-82.

21. Bhatti AB, Ali F, Satti SA (2014) Prolonged QTc Interval is an electrophysiologica hallmark of cirrhotic cardiomyopathy. Open Journal of Internal Medicine 4: 33-39.

22. Puthumana L, Chaudhry V, Thuluvath PJ (2001) Prolonged QTc interval and its relationship to autonomic cardiovascular reflexes in patients with cirrhosis. Journal of hepatology 35: 733-738.

23. Thomopoulos K, Tziakas D, Ritis K, Dalla V, Kotsiou S, et al. (2007) Prolongation of the QTc interval in patients with cirrhosis.

24. Moller S, Henriksen JH (2010) Cirrhotic cardiomyopathy. Journal of Hepatology 53 179-190.

25. Day CP, James OF, Butler TJ, Campbell RW (1993) QT prolongation and sudden cardiac death in patients with alcoholic liver disease. Lancet 341: 1423-1428.

26. Makkar RR, Fromm BS, Steinman RT, Meissner MD, Lehmann MH (1993) Female gender as a risk factor for torsades de pointes associated with cardiovascular drugs. JAMA 270: 2590-2597.

27. Zuberi BF, Ahmed S, Faisal N, Afsar S, Memon AR, et al. (2007) Comparison of heart rate and QTc duration in patients of cirrhosis of liver with non-cirrhotic controls Journal of the College of Physicians and Surgeons--Pakistan 17: 69-71.

28. Li L, Liu HR, Shu JL, Xi XP, Wang Y (2007) Clinical investigation of Q-T prolongation in hepatic cirrhosis. Zhonghua Yi Xue Za Zhi 87: 2717-2718.

29. Zamirian M, Tavassoli M, Aghasadeghi K (2012) Corrected QT interval and QT dispersion in cirrhotic patients before and after liver transplantation. Archives of Iranian Medicine 15: 375-377.

30. Kosar F, Ates F, Sahin I, Karincaoglu M (2007) Yildirim B. QT interval analysis in patients with chronic liver disease: a prospective study. Angiology 58: 218-224.

31. Nasr GMA, Eldin MM, Ragheb M (2008) Systolic and diastolic functions, QT interval and myocardial perfusion imaging in post-viral cirrhosis with and without ascites. Heart Mirror Journal 2: 28-35.

32. Lossintzer D, Steen H, Zehn A (2010) Myocardial late gadolinium enhancement cardiovascular magnetic resonance in patients with cirrhosis. Journal of Cardiovascular Magnetic Resonance 12: 1-10.

33. Lehmann MH (1997) QT prolongation in end-stage liver disease: a result of altered sex hormone metabolism? Hepatology 26: 244.

34. Genovesi S, Prata Pizzala DM, Pozzi M, Ratti L, Milanese M, et al. (2009) QT interval prolongation and decreased heart rate variability in cirrhotic patients: relevance of hepatic venous pressure gradient and serum calcium. Clinical Science 116: 851-859.

35. Salari A, Shafaghi A, Ofoghi M, Saeidinia A, Mansour-Ghanaei F (2013) Diastolic dysfunction and severity of cirrhosis in nonalcoholic cirrhotic patients. International Journal of Hepatology.

Copyright: $\mathbb{C} 2020$ Saeidinia A. This is an open-access article distributed under the terms of the Creative Commons Attribution License, which permits unrestricted use, distribution, and reproduction in any medium, provided the original author and source are credited. 